\title{
Coefficient Estimates and Other Properties for a Class of Spirallike Functions Associated with a Differential Operator
}

\author{
Halit Orhan, ${ }^{1}$ Dorina Răducanu, ${ }^{2}$ Murat Çağlar, ${ }^{1}$ and Mustafa Bayram ${ }^{3}$ \\ ${ }^{1}$ Department of Mathematics, Faculty of Science, Atatürk University, 25240 Erzurum, Turkey \\ ${ }^{2}$ Department of Mathematics, Faculty of Mathematics and Computer Science, Transylvania University of Braşov, \\ Iuliu Maniu 50, 50091 Brașov, Romania \\ ${ }^{3}$ Department of Mathematical Engineering, Faculty of Chemical and Metallurgical Engineering, \\ Yildiz Technical University, Davutpasa, 34210 Istanbul, Turkey \\ Correspondence should be addressed to Halit Orhan; orhanhalit607@gmail.com
}

Received 17 February 2013; Accepted 16 May 2013

Academic Editor: Adem Kılıçman

Copyright (C) 2013 Halit Orhan et al. This is an open access article distributed under the Creative Commons Attribution License, which permits unrestricted use, distribution, and reproduction in any medium, provided the original work is properly cited.

For $0 \leq \eta<1,0 \leq \lambda<1,-\pi / 2<\gamma<\pi / 2,0 \leq \beta \leq \alpha$, and $m \in \mathbb{N} \cup\{0\}$, a new class $S_{\alpha, \beta}^{m}(\eta, \gamma, \lambda)$ of analytic functions defined by means of the differential operator $D_{\alpha, \beta}^{m}$ is introduced. Our main object is to provide sharp upper bounds for Fekete-Szegö problem in $S_{\alpha, \beta}^{m}(\eta, \gamma, \lambda)$. We also find sufficient conditions for a function to be in this class. Some interesting consequences of our results are pointed out.

\section{Introduction}

Let $\mathscr{A}$ denote the class of functions $f$ of the form

$$
f(z)=z+\sum_{n=2}^{\infty} a_{n} z^{n}
$$

which are analytic in the open unit disk $\mathscr{U}=\{z \in \mathbb{C}:|z|<1\}$.

Let $\mathcal{S}$ denote the subclass of $\mathscr{A}$ consisting of functions that are univalent in $\mathcal{U}$.

A function $f \in \mathscr{A}$ is said to be in the class of $\gamma$-spirallike functions of order $\lambda$ in $\mathcal{U}$, denoted by $\mathcal{S}^{*}(\gamma, \lambda)$, if

$$
\Re\left(e^{i \gamma} \frac{z f^{\prime}(z)}{f(z)}\right)>\lambda \cos \gamma, \quad z \in \mathcal{U},
$$

for $0 \leq \lambda<1$ and some real $\gamma$ with $|\gamma|<\pi / 2$.

The class $\mathcal{S}^{*}(\gamma, \lambda)$ was studied by Libera [1] and Keogh and Merkes [2].

Note that $\mathcal{S}^{*}(\gamma, 0)$ is the class of spirallike functions introduced by Špaček [3], $\mathcal{S}^{*}(0, \lambda)=\mathcal{S}^{*}(\lambda)$ is the class of starlike functions of order $\lambda$, and $\mathcal{S}^{*}(0,0)=\mathcal{S}^{*}$ is the familiar class of starlike functions.
For the constants $\lambda, \gamma$ with $0 \leq \lambda<1$ and $|\gamma|<\pi / 2$, denote

$$
p_{\lambda, \gamma}(z)=\frac{1+e^{-i \gamma}\left(e^{-i \gamma}-2 \lambda \cos \gamma\right) z}{1-z}, \quad z \in \mathscr{U} .
$$

The function $p_{\lambda, \gamma}(z)$ maps the open unit disk onto the halfplane $H_{\lambda, \gamma}=\left\{z \in \mathbb{C}: \mathfrak{R}\left(e^{i \gamma} z\right)>\lambda \cos \gamma\right\}$. If

$$
p_{\lambda, \gamma}(z)=1+\sum_{n=1}^{\infty} p_{n} z^{n}
$$

then it is easy to check that

$$
p_{n}=2 e^{-i \gamma}(1-\lambda) \cos \gamma, \quad \forall n \geq 1 .
$$

For $f \in \mathscr{A}$ given by (1) and $g \in \mathscr{A}$ given by

$$
g(z)=z+\sum_{n=2}^{\infty} b_{n} z^{n}
$$

the Hadamard product (or convolution), denoted by $f * g$, is defined by

$$
(f * g)(z)=z+\sum_{n=2}^{\infty} a_{n} b_{n} z^{n}, \quad z \in \mathcal{U}
$$


Denote by $\mathscr{B}$ the family of all analytic functions $w(z)$ that satisfy the conditions $w(0)=0$ and $|w(z)|<1, z \in \mathcal{U}$.

A function $f \in \mathscr{A}$ is said to be subordinate to a function $g \in \mathscr{A}$, written $f \prec g$, if there exists a function $w \in \mathscr{B}$ such that $f(z)=g(w(z)), z \in \mathcal{U}$.

A classical theorem of Fekete and Szegö (see [4]) states that if $f \in \mathcal{S}$ is given by (1), then

$$
\left|a_{3}-\mu a_{2}^{2}\right| \leq \begin{cases}3-4 \mu, & \text { if } \mu \leq 0, \\ 1+2 \exp \left(\frac{-2 \mu}{1-\mu}\right), & \text { if } 0 \leq \mu \leq 1, \\ 4 \mu-3, & \text { if } \mu \geq 1 .\end{cases}
$$

This inequality is sharp in the sense that for each $\mu$ there exists a function in $\delta$ such that the equality holds. Later Pfluger (see [5]) has considered the same problem but for complex values of $\mu$. The problem of finding sharp upper bounds for the functional $\left|a_{3}-\mu a_{2}^{2}\right|$ for different subclasses of $\mathscr{A}$ is known as the Fekete-Szegö problem. Over the years, this problem has been investigated by many authors including [6-12].

For a function $f \in \mathscr{A}$, we consider the following differential operator introduced by Răducanu and Orhan [13]:

(i) $D_{\alpha, \beta}^{0} f(z)=f(z)$,

(ii) $D_{\alpha, \beta}^{1} f(z)=D_{\alpha, \beta} f(z)=\alpha \beta z^{2} f^{\prime \prime}(z)+(\alpha-\beta) z f^{\prime}(z)+$ $(1-\alpha+\beta) f(z)$,

(iii) $D_{\alpha, \beta}^{m} f(z)=D_{\alpha, \beta}\left(D_{\alpha, \beta}^{m-1} f(z)\right), z \in \mathcal{U}$,

where $0 \leq \beta \leq \alpha$ and $m \in \mathbb{N}_{0}=\{0,1, \ldots\}$.

If the function $f$ is given by (1), then, from the definition of the operator $D_{\alpha, \beta}^{m} f$, it is easy to observe that

$$
D_{\alpha, \beta}^{m} f(z)=z+\sum_{n=2}^{\infty} \Phi_{n}(\alpha, \beta, m) a_{n} z^{n}
$$

where

$$
\Phi_{n}(\alpha, \beta, m)=[1+(\alpha \beta n+\alpha-\beta)(n-1)]^{m}, \quad n \geq 2 .
$$

It should be remarked that the operator $D_{\alpha, \beta}^{m} f$ generalizes other differential operators considered earlier. For $f \in \mathscr{A}$, we have

(i) $D_{1,0}^{m} f(z)=D^{m} f(z)$, the operator introduced by Sălăgean [14];

(ii) $D_{\alpha, 0}^{m} f(z)=D_{\alpha}^{m} f(z)$, the operator studied by AlOboudi [15].

In view of (9), $D_{\alpha, \beta}^{m} f(z)$ can be written in terms of convolution as

$$
D_{\alpha, \beta}^{m} f(z)=\left(g_{\alpha, \beta} * f\right)(z), \quad z \in \mathcal{U},
$$

where

$$
g_{\alpha, \beta}(z)=z+\sum_{n=2}^{\infty} \Phi_{n}(\alpha, \beta, m) z^{n}, \quad z \in \mathcal{U}
$$

Define the function $g_{\alpha, \beta}^{(-1)}$ such that

$$
g_{\alpha, \beta}^{(-1)}(z) * g_{\alpha, \beta}(z)=\frac{z}{1-z}, \quad z \in \mathcal{U} .
$$

It is easy to observe that

$$
f(z)=g_{\alpha, \beta}^{(-1)}(z) * D_{\alpha, \beta}^{m} f(z) .
$$

Making use of the differential operator $D_{\alpha, \beta}^{m} f$, we define the following class of functions.

Definition 1. For $0 \leq \eta<1,0 \leq \lambda<1$, and $|\gamma|<\pi / 2$, denote by $\mathcal{S}_{\alpha, \beta}^{m}(\eta, \gamma, \lambda)$ the class of functions $f \in \mathscr{A}$ which satisfy the condition

$$
\mathfrak{R}\left(e^{i \gamma} \frac{z\left(D_{\alpha, \beta}^{m} f(z)\right)^{\prime}}{(1-\eta) D_{\alpha, \beta}^{m} f(z)+\eta z\left(D_{\alpha, \beta}^{m} f(z)\right)^{\prime}}\right)>\lambda \cos \gamma,
$$

$$
z \in \mathcal{U}
$$

The class $\mathcal{S}_{\alpha, \beta}^{m}(\eta, \gamma, \lambda)$ contains as particular cases the following classes of functions:

$$
\begin{gathered}
\mathcal{S}_{\alpha, \beta}^{0}(0, \gamma, \lambda)=\mathcal{\delta}^{*}(\gamma, \lambda), \\
\mathcal{S}_{\alpha, \beta}^{0}(0, \gamma, 0)=\mathcal{S}^{*}(\gamma), \quad \mathcal{\delta}_{\alpha, \beta}^{0}(0,0,0)=\mathcal{S}^{*} .
\end{gathered}
$$

Also, the class $\delta_{\alpha, \beta}^{0}(\eta, \gamma, \lambda)$ consists of functions $f \in \mathscr{A}$ satisfying the inequality

$$
\mathfrak{R}\left(e^{i \gamma} \frac{z f^{\prime}(z)}{(1-\eta) f(z)+\eta z f^{\prime}(z)}\right)>\lambda \cos \gamma, \quad z \in \mathcal{U} .
$$

An analogous of the class $\delta_{\alpha, \beta}^{0}(\eta, \gamma, \lambda)$ has been recently studied by Murugusundaramoorthy [16].

The main object of this paper is to obtain sharp upper bounds for the Fekete-Szegö problem for the class $\mathcal{S}_{\alpha, \beta}^{m}(\eta$, $\gamma, \lambda)$. We also find sufficient conditions for a function to be in this class.

\section{Membership Characterizations}

In this section, we obtain several sufficient conditions for a function $f \in \mathscr{A}$ to be in the class $\mathcal{S}_{\alpha, \beta}^{m}(\eta, \gamma, \lambda)$.

Theorem 2. Let $f \in \mathscr{A}$, and let $\delta$ be a real number with $0 \leq$ $\delta<1$. If

$$
\left|\frac{z\left(D_{\alpha, \beta}^{m} f(z)\right)^{\prime}}{(1-\eta) D_{\alpha, \beta}^{m} f(z)+\eta z\left(D_{\alpha, \beta}^{m} f(z)\right)^{\prime}}-1\right| \leq 1-\delta, \quad z \in \mathcal{U},
$$

then $f \in \mathcal{S}_{\alpha, \beta}^{m}(\eta, \gamma, \lambda)$ provided that

$$
|\gamma| \leq \cos ^{-1}\left(\frac{1-\delta}{1-\lambda}\right)
$$


Proof. From (18), it follows that

$$
\frac{z\left(D_{\alpha, \beta}^{m} f(z)\right)^{\prime}}{(1-\eta) D_{\alpha, \beta}^{m} f(z)+\eta z\left(D_{\alpha, \beta}^{m} f(z)\right)^{\prime}}=1+(1-\delta) w(z),
$$

where $w(z) \in \mathscr{B}$. We have

$$
\begin{aligned}
& \mathfrak{R}\left(e^{i \gamma} \frac{z\left(D_{\alpha, \beta}^{m} f(z)\right)^{\prime}}{(1-\eta) D_{\alpha, \beta}^{m} f(z)+\eta z\left(D_{\alpha, \beta}^{m} f(z)\right)^{\prime}}\right) \\
& \quad=\Re\left[e^{i \gamma}(1+(1-\delta) w(z))\right] \\
& =\cos \gamma+(1-\delta) \mathfrak{R}\left(e^{i \gamma} w(z)\right) \\
& \quad \geq \cos \gamma-(1-\delta)\left|e^{i \gamma} w(z)\right| \\
& >\cos \gamma-(1-\delta) \geq \lambda \cos \gamma,
\end{aligned}
$$

provided that $|\gamma| \leq \cos ^{-1}((1-\delta) /(1-\lambda))$. Thus, the proof is completed.

If in Theorem 2 we take $\delta=1-(1-\lambda) \cos \gamma$, we will obtain the following result.

Corollary 3. Let $f \in \mathscr{A}$. If

$$
\left|\frac{z\left(D_{\alpha, \beta}^{m} f(z)\right)^{\prime}}{(1-\eta) D_{\alpha, \beta}^{m} f(z)+\eta z\left(D_{\alpha, \beta}^{m} f(z)\right)^{\prime}}-1\right| \leq(1-\lambda) \cos \gamma
$$

then $f \in \mathcal{S}_{\alpha, \beta}^{m}(\eta, \gamma, \lambda)$.

A sufficient condition for a function $f \in \mathscr{A}$ to be in the class $\mathcal{S}_{\alpha, \beta}^{m}(\eta, \gamma, \lambda)$, in terms of coefficients inequality, is obtained in the next theorem.

Theorem 4. If a function $f \in \mathscr{A}$ given by (1) satisfies the inequality

$$
\begin{aligned}
\sum_{n=2}^{\infty}[ & (1-\eta)(n-1) \sec \gamma+(1-\lambda)(1+\eta(n-1))] \\
& \times \Phi_{n}(\alpha, \beta, m)\left|a_{n}\right| \leq 1-\lambda,
\end{aligned}
$$

where $0 \leq \eta<1,0 \leq \lambda<1,|\gamma|<\pi / 2$, and $\Phi_{n}(\alpha, \beta, m)$ is defined by (10), then it belongs to the class $\mathcal{S}_{\alpha, \beta}^{m}(\eta, \gamma, \lambda)$.
Proof. In virtue of Corollary 3 , it suffices to show that the condition (22) is satisfied. We have

$$
\begin{aligned}
& \left|\frac{z\left(D_{\alpha, \beta}^{m} f(z)\right)^{\prime}}{(1-\eta) D_{\alpha, \beta}^{m} f(z)+\eta z\left(D_{\alpha, \beta}^{m} f(z)\right)^{\prime}}-1\right| \\
& \quad=(1-\eta)\left|\frac{\sum_{n=2}^{\infty}(n-1) \Phi_{n}(\alpha, \beta, m) a_{n} z^{n-1}}{1+\sum_{n=2}^{\infty}(1-\eta+\eta n) \Phi_{n}(\alpha, \beta, m) a_{n} z^{n-1}}\right| \\
& <(1-\eta) \frac{\sum_{n=2}^{\infty}(n-1) \Phi_{n}(\alpha, \beta, m)\left|a_{n}\right|}{1-\sum_{n=2}^{\infty}(1-\eta+\eta n) \Phi_{n}(\alpha, \beta, m)\left|a_{n}\right|} .
\end{aligned}
$$
if

The last expression is bounded previously by $(1-\lambda) \cos \gamma$,

$$
\begin{aligned}
& \sum_{n=2}^{\infty}(1-\eta)(n-1) \Phi_{n}(\alpha, \beta, m)\left|a_{n}\right| \\
& \quad \leq(1-\lambda) \cos \gamma\left(1-\sum_{n=2}^{\infty}(1-\eta+\eta n) \Phi_{n}(\alpha, \beta, m)\left|a_{n}\right|\right),
\end{aligned}
$$

which is equivalent to

$$
\begin{aligned}
& \sum_{n=2}^{\infty}[(1-\eta)(n-1) \sec \gamma+(1-\lambda)(1+\eta(n-1))] \\
& \quad \times \Phi_{n}(\alpha, \beta, m)\left|a_{n}\right| \leq 1-\lambda .
\end{aligned}
$$

For special values of $m, \eta, \gamma$, and $\lambda$, from Theorem 4 , we can derive the following sufficient conditions for a function $f \in \mathscr{A}$ to be in the classes $\mathcal{S}_{\alpha, \beta}^{0}(\eta, \gamma, \lambda), \mathcal{\delta}_{\alpha, \beta}^{0}(0, \gamma, \lambda)=\mathcal{S}^{*}(\gamma$, $\lambda)$, and $\delta_{\alpha, \beta}^{0}(0, \gamma, 0)=\mathcal{S}^{*}(\gamma)$, respectively.

Corollary 5. Let $f \in \mathscr{A}$. If

$$
\sum_{n=2}^{\infty}[(1-\eta)(n-1) \sec \gamma+(1-\lambda)(1+\eta(n-1))]\left|a_{n}\right|
$$

$$
\leq 1-\lambda
$$

where $0 \leq \eta<1,0 \leq \lambda<1$, and $|\gamma|<\pi / 2$, then $f \in \mathcal{S}_{\alpha, \beta}^{0}(\eta$, $\gamma, \lambda)$.

Corollary 6 (see [17]). Let $f \in \mathscr{A}$. If

$$
\sum_{n=2}^{\infty}[(n-1) \sec \gamma+1-\lambda]\left|a_{n}\right| \leq 1-\lambda,
$$

where $0 \leq \lambda<1,|\gamma|<\pi / 2$, then $f \in \mathcal{S}^{*}(\gamma, \lambda)$.

Corollary 7 (see [18]). Let $f \in \mathscr{A}$. If

$$
\sum_{n=2}^{\infty}[1+(n-1) \sec \gamma]\left|a_{n}\right| \leq 1,
$$

where $|\gamma|<\pi / 2$, then $f \in \mathcal{S}^{*}(\gamma)$. 
A necessary and sufficient condition for a function to be in the class $\mathcal{S}_{\alpha, \beta}^{m}(\eta, \gamma, \lambda)$ can be given in terms of integral representation.

Theorem 8. A function $f \in \mathscr{A}$ is in the class $\delta_{\alpha, \beta}^{m}(\eta, \gamma, \lambda)$ if and only if there exists $w \in \mathscr{B}$ such that

$$
\begin{array}{r}
f(z)=g_{\alpha, \beta}^{(-1)}(z) * z \exp \left(\int_{0}^{z}\left[\frac{p_{\lambda, \gamma}(w(\zeta))-1}{1-\eta p_{\lambda, \gamma}(w(\zeta))}\right] \frac{d \zeta}{\zeta}\right), \\
z \in \mathcal{U}
\end{array}
$$

where $p_{\lambda, \gamma}(z)$ and $g_{\alpha, \beta}^{(-1)}(z)$ are defined by (3) and (13), respectively.

Proof. In virtue of (15), $f \in \mathcal{S}_{\alpha, \beta}^{m}(\eta, \gamma, \lambda)$ if and only if there exists $w \in \mathscr{B}$ such that

$$
\frac{z\left(D_{\alpha, \beta}^{m} f(z)\right)^{\prime}}{(1-\eta) D_{\alpha, \beta}^{m} f(z)+\eta z\left(D_{\alpha, \beta}^{m} f(z)\right)^{\prime}}=p_{\lambda, \gamma}(w(z)) .
$$

From the last equality, we obtain

$$
D_{\alpha, \beta}^{m} f(z)=z \exp \left(\int_{0}^{z}\left[\frac{p_{\lambda, \gamma}(w(\zeta))-1}{1-\eta p_{\lambda, \gamma}(w(\zeta))}\right] \frac{d \zeta}{\zeta}\right)
$$

Making use of (14) and (32), we have

$$
\begin{array}{r}
f(z)=g_{\alpha, \beta}^{(-1)}(z) * z \exp \left(\int_{0}^{z}\left[\frac{p_{\lambda, \gamma}(w(\zeta))-1}{1-\eta p_{\lambda, \gamma}(w(\zeta))}\right] \frac{d \zeta}{\zeta}\right) \\
z \in \mathcal{U}
\end{array}
$$

and thus, the proof is completed.

For $0 \leq \theta \leq 2 \pi, 0 \leq \tau \leq 1$, define the function

$$
\begin{aligned}
& \Psi(z, \theta, \tau) \\
& \quad=g_{\alpha, \beta}^{(-1)}(z) \\
& \quad * z \exp \left(\int_{0}^{z}\left[\frac{p_{\lambda, \gamma}\left(e^{i \theta} \zeta(\zeta+\tau) /(1+\tau \zeta)\right)-1}{1-\eta p_{\lambda, \gamma}\left(e^{i \theta} \zeta(\zeta+\tau) /(1+\tau \zeta)\right)}\right] \frac{d \zeta}{\zeta}\right),
\end{aligned}
$$

where $p_{\lambda, \gamma}(z)$ and $g_{\alpha, \beta}^{(-1)}(z)$ are defined by (3) and (13), respectively.

In virtue of Theorem 8 , the function $\Psi(z, \theta, \tau)$ belongs to the class $\mathcal{S}_{\alpha, \beta}^{m}(\eta, \gamma, \lambda)$. Note that $\Psi(z, 0,0)$ is an odd function.

\section{The Fekete-Szegö Problem}

In order to obtain sharp upper bounds for the Fekete-Szegö functional for the class $\mathcal{S}_{\alpha, \beta}^{m}(\eta, \gamma, \lambda)$, the following lemma is required (see, e.g., [19, page 108]).
Lemma 9. Let the function $w \in \mathscr{B}$ be given by

$$
w(z)=\sum_{n=1}^{\infty} w_{n} z^{n}, \quad z \in \mathcal{U}
$$

Then

$$
\left|w_{1}\right| \leq 1, \quad\left|w_{2}\right| \leq 1-\left|w_{1}\right|^{2},
$$

$\left|w_{2}-s w_{1}^{2}\right| \leq \max \{1,|s|\}, \quad$ for any complex number $s$.

The functions $w(z)=z$ and $w(z)=z^{2}$, or one of their rotations, show that both inequalities (36) and (37) are sharp.

First we obtain sharp upper bounds for the Fekete-Szegö functional $\left|a_{3}-\mu a_{2}^{2}\right|$ with $\mu$ real parameter.

Theorem 10. Let $f \in \mathcal{S}_{\alpha, \beta}^{m}(\eta, \gamma, \lambda)$ be given by (1), and let $\mu$ be a real number. Then

$$
\begin{aligned}
& \left|a_{3}-\mu a_{2}^{2}\right| \\
& \left\{\begin{array}{l}
\frac{(1-\lambda) \cos \gamma}{(1-\eta)^{2} \Phi_{3}(\alpha, \beta, m)} \\
\times[\eta+3-2 \lambda(1+\eta) \\
\left.\quad-\mu \frac{4(1-\lambda) \Phi_{3}(\alpha, \beta, m)}{\Phi_{2}^{2}(\alpha, \beta, m)}\right], \\
\frac{(1-\lambda) \cos \gamma}{(1-\eta) \Phi_{3}(\alpha, \beta, m)}, \\
\frac{(1-\lambda) \cos \gamma}{(1-\eta)^{2} \Phi_{3}(\alpha, \beta, m)} \\
\times\left[\mu \frac{4(1-\lambda) \Phi_{3}(\alpha, \beta, m)}{\Phi_{2}^{2}(\alpha, \beta, m)}\right. \\
+2 \lambda(1+\eta)-\eta-3],
\end{array} \quad \text { if } \sigma_{1} \leq \mu \leq \sigma_{2},\right.
\end{aligned}
$$

where

$$
\begin{gathered}
\sigma_{1}=(1+\eta) \frac{\Phi_{2}^{2}(\alpha, \beta, m)}{2 \Phi_{3}(\alpha, \beta, m)}, \\
\sigma_{2}=\frac{2-\lambda(1+\eta)}{1-\lambda} \frac{\Phi_{2}^{2}(\alpha, \beta, m)}{2 \Phi_{3}(\alpha, \beta, m)},
\end{gathered}
$$

and $\Phi_{2}(\alpha, \beta, m), \Phi_{3}(\alpha, \beta, m)$ are defined by (10) with $n=2$ and $n=3$, respectively.

All estimates are sharp. 
Proof. Suppose that $f \in \mathcal{S}_{\alpha, \beta}^{m}(\eta, \gamma, \lambda)$ is given by (1). Then, from the definition of the class $\mathcal{S}_{\alpha, \beta}^{m}(\eta, \gamma, \lambda)$, there exist $w \in$ $\mathscr{B}, w(z)=w_{1} z+w_{2} z^{2}+w_{3} z^{3}+\cdots$ such that

$$
\frac{z\left(D_{\alpha, \beta}^{m} f(z)\right)^{\prime}}{(1-\eta) D_{\alpha, \beta}^{m} f(z)+\eta z\left(D_{\alpha, \beta}^{m} f(z)\right)^{\prime}}=p_{\lambda, \gamma}(w(z)),
$$$$
z \in \mathcal{U}
$$

Set $p_{\lambda, \gamma}(z)=1+p_{1} z+p_{2} z^{2}+p_{3} z^{3}+\cdots$. Equating the coefficients of $z$ and $z^{2}$ on both sides of (41), we obtain

$$
\begin{gathered}
a_{2}=\frac{p_{1} w_{1}}{(1-\eta) \Phi_{2}(\alpha, \beta, m)}, \\
a_{3}=\frac{1}{2(1-\eta) \Phi_{3}(\alpha, \beta, m)}\left[\left(\frac{1+\eta}{1-\eta} p_{1}^{2}+p_{2}\right) w_{1}^{2}+p_{1} w_{2}\right] .
\end{gathered}
$$

From (5), we have $p_{1}=p_{2}=2 e^{-i \gamma}(1-\lambda) \cos \gamma$, and thus we obtain

$$
\begin{gathered}
a_{2}=\frac{2 e^{-i \gamma}(1-\lambda) \cos \gamma}{(1-\eta) \Phi_{2}(\alpha, \beta, m)} w_{1}, \\
a_{3}=\frac{e^{-i \gamma}(1-\lambda) \cos \gamma}{(1-\eta) \Phi_{3}(\alpha, \beta, m)} \\
\times\left[\left(2 e^{-i \gamma}(1-\lambda) \cos \gamma \frac{1+\eta}{1-\eta}+1\right) w_{1}^{2}+w_{2}\right] .
\end{gathered}
$$

It follows that

$$
\begin{aligned}
& \mid a_{3}-\mu a_{2}^{2} \mid \\
& \leq \frac{(1-\lambda) \cos \gamma}{(1-\eta) \Phi_{3}(\alpha, \beta, m)} \\
& \quad \times\left\{\left|\frac{2 e^{-i \gamma}(1-\lambda) \cos \gamma}{1-\eta}\left(1+\eta-\mu \frac{2 \Phi_{3}(\alpha, \beta, m)}{\Phi_{2}^{2}(\alpha, \beta, m)}\right)+1\right|\right. \\
&\left.\quad \times\left|w_{1}\right|^{2}+\left|w_{2}\right|\right\} .
\end{aligned}
$$

Making use of Lemma 9 (36), we have

$$
\begin{aligned}
& \left|a_{3}-\mu a_{2}^{2}\right| \\
& \leq \frac{(1-\lambda) \cos \gamma}{(1-\eta) \Phi_{3}(\alpha, \beta, m)} \\
& \quad \times\left\{1+\left[\mid \frac{2 e^{-i \gamma}(1-\lambda) \cos \gamma}{1-\eta}\right.\right. \\
& \left.\quad \times\left(1+\eta-\mu \frac{2 \Phi_{3}(\alpha, \beta, m)}{\Phi_{2}^{2}(\alpha, \beta, m)}\right)+1 \mid-1\right] \\
& \left.\quad \times\left|w_{1}\right|^{2}\right\}
\end{aligned}
$$

or

$$
\begin{aligned}
\left|a_{3}-\mu a_{2}^{2}\right| \leq & \frac{(1-\lambda) \cos \gamma}{(1-\eta) \Phi_{3}(\alpha, \beta, m)} \\
& \times\left[1+\left(\sqrt{1+M(2+M) \cos ^{2} \gamma}-1\right)\left|w_{1}\right|^{2}\right]
\end{aligned}
$$

where

$$
M=\frac{2(1-\lambda)}{1-\eta}\left(1+\eta-\mu \frac{2 \Phi_{3}(\alpha, \beta, m)}{\Phi_{2}^{2}(\alpha, \beta, m)}\right) .
$$

Denote

$$
F(x, y)=1+\left(\sqrt{1+M(2+M) x^{2}}-1\right) y^{2},
$$

where $x=\cos \gamma, y=\left|w_{1}\right|$, and $(x, y) \in[0,1] \times[0,1]$.

Simple calculation shows that the function $F(x, y)$ does not have a local maximum at any interior point of the open rectangle $(0,1) \times(0,1)$. Thus, the maximum must be attained at a boundary point. Since $F(x, 0)=1, F(0, y)=1$, and $F(1,1)=|1+M|$, it follows that the maximal value of $F(x, y)$ may be $F(0,0)=1$ or $F(1,1)=|1+M|$.

Therefore, from (46), we obtain

$$
\left|a_{3}-\mu a_{2}^{2}\right| \leq \frac{(1-\lambda) \cos \gamma}{(1-\eta) \Phi_{3}(\alpha, \beta, m)} \max \{1,|1+M|\},
$$

where $M$ is given by (47).

Consider first the case $|1+M| \geq 1$. If $\mu \leq \sigma_{1}$, where $\sigma_{1}$ is given by (39), then $M \geq 0$, and from (49), we obtain

$$
\begin{aligned}
\mid a_{3}- & \mu a_{2}^{2} \mid \\
\leq & \frac{(1-\lambda) \cos \gamma}{(1-\eta)^{2} \Phi_{3}(\alpha, \beta, m)} \\
& \times\left[\eta+3-2 \lambda(1+\eta)-\mu \frac{4(1-\lambda) \Phi_{3}(\alpha, \beta, m)}{\Phi_{2}^{2}(\alpha, \beta, m)}\right],
\end{aligned}
$$

which is the first part of the inequality (38). If $\mu \geq \sigma_{2}$, where $\sigma_{2}$ is given by (40), then $M \leq-2$, and it follows from (49) that

$$
\begin{aligned}
& \mid a_{3}-\mu a_{2}^{2} \mid \\
& \leq \frac{(1-\lambda) \cos \gamma}{(1-\eta)^{2} \Phi_{3}(\alpha, \beta, m)} \\
& \quad \times\left[\mu \frac{4(1-\lambda) \Phi_{3}(\alpha, \beta, m)}{\Phi_{2}^{2}(\alpha, \beta, m)}+2 \lambda(1+\eta)-\eta-3\right],
\end{aligned}
$$

and this is the third part of (38).

Next, suppose that $\sigma_{1} \leq \mu \leq \sigma_{2}$. Then, $|1+M| \leq 1$, and thus, from (49), we obtain

$$
\left|a_{3}-\mu a_{2}^{2}\right| \leq \frac{(1-\lambda) \cos \gamma}{(1-\eta) \Phi_{3}(\alpha, \beta, m)},
$$

which is the second part of the inequality (38). 
In view of Lemma 9, the results are sharp for $w(z)=z$ and $w(z)=z^{2}$ or one of their rotations. From (41), we obtain that the extremal functions are $\Psi(z, \theta, 1)$ and $\Psi(z, \theta, 0)$ defined by (34) with $\tau=1$ and $\tau=0$.

Next, we consider the Fekete-Szegö problem for the class $\mathcal{S}_{\alpha, \beta}^{m}(\eta, \gamma, \lambda)$ with $\mu$ complex parameter.

Theorem 11. Let $f \in \mathcal{S}_{\alpha, \beta}^{m}(\eta, \gamma, \lambda)$ be given by (1), and let $\mu$ be a complex number. Then,

$$
\begin{aligned}
& \left|a_{3}-\mu a_{2}^{2}\right| \\
& \leq \frac{(1-\lambda) \cos \gamma}{(1-\eta) \Phi_{3}(\alpha, \beta, m)} \\
& \times \max \left\{1, \mid \frac{2(1-\lambda) \cos \gamma}{1-\eta}\right. \\
& \left.\times\left(\mu \frac{2 \Phi_{3}(\alpha, \beta, m)}{\Phi_{2}^{2}(\alpha, \beta, m)}-1-\eta\right)-e^{i \gamma} \mid\right\} .
\end{aligned}
$$

The result is sharp.

Proof. Assume that $f \in \mathcal{S}_{\alpha, \beta}^{m}(\eta, \gamma, \lambda)$. Making use of (43), we obtain

$$
\begin{aligned}
& \left|a_{3}-\mu a_{2}^{2}\right| \\
& \leq \frac{(1-\lambda) \cos \gamma}{(1-\eta) \Phi_{3}(\alpha, \beta, m)} \\
& \times \mid w_{2}-\left[\frac{2 e^{-i \gamma}(1-\lambda) \cos \gamma}{1-\eta}\right. \\
& \left.\times\left(\mu \frac{2 \Phi_{3}(\alpha, \beta, m)}{\Phi_{2}^{2}(\alpha, \beta, m)}-1-\eta\right)-1\right] w_{1}^{2} \mid .
\end{aligned}
$$

The inequality (53) follows as an application of Lemma 9 (37) with

$$
s=\frac{2 e^{-i \gamma}(1-\lambda) \cos \gamma}{1-\eta}\left(\mu \frac{2 \Phi_{3}(\alpha, \beta, m)}{\Phi_{2}^{2}(\alpha, \beta, m)}-1-\eta\right)-1
$$

The functions $\Psi(z, \theta, 1)$ and $\Psi(z, \theta, 0)$ defined by (34) with $\tau=1$ and $\tau=0$ show that the inequality (53) is sharp.

Our Theorems 10 and 11 include several various results for special values of $m, \eta, \gamma$, and $\lambda$. For example, taking $m=\eta=\gamma=\lambda=0$, in Theorem 10, we obtain the FeketeSzegö inequalities for the class $\mathcal{S}^{*}$ (see $[2,11]$ ). The special case $m=\eta=\lambda=0$ leads to the Fekete-Szegö inequalities for the class $\mathcal{S}^{*}(\gamma)$ (see [2]). The Fekete-Szegö inequalities for the class $\mathcal{S}^{*}(\gamma, \lambda)$ (see [2]) are also included in Theorems 10 and 11.

\section{Acknowledgments}

The authors thank the referees for their valuable suggestions to improve the paper. The first and third authors' research was supported by Atatürk University Rectorship under "The Scientific and Research Project of Atatürk University," Project no. $2012 / 173$.

\section{References}

[1] R. J. Libera, "Univalent $\alpha$-spiral functions," Canadian Journal of Mathematics., vol. 19, pp. 725-733, 1967.

[2] F. R. Keogh and E. P. Merkes, "A coefficient inequality for certain classes of analytic functions," Proceedings of the American Mathematical Society, vol. 20, pp. 8-12, 1969.

[3] L. Špaček, "Contribution à la théorie des functions univalents," Casopis Pro Pestování Matematiky A Fysiky, vol. 62, no. 2, pp. 12-19, 1932.

[4] M. Fekete and G. Szegö, "Eine bemerkung uber ungerade schlichte funktionen," The Journal of the London Mathematical Society, vol. 8, no. 2, pp. 85-89.

[5] A. Pfluger, "The Fekete-Szego" inequality for complex parameters," Complex Variables. Theory and Application, vol. 7, no. 1-3, pp. 149-160, 1986.

[6] E. Deniz and H. Orhan, "The Fekete-Szegö problem for a generalized subclass of analytic functions," Kyungpook Mathematical Journal, vol. 50, no. 1, pp. 37-47, 2010.

[7] E. Deniz, M. Çağlar, and H. Orhan, "The Fekete-Szegö problem for a class of analytic functions defined by Dziok-Srivastava operator," Kodai Mathematical Journal, vol. 35, no. 3, pp. 439462, 2012.

[8] A. K. Mishra and P. Gochhayat, "Fekete-Szegö problem for a class defined by an integral operator," Kodai Mathematical Journal, vol. 33, no. 2, pp. 310-328, 2010.

[9] H. Orhan, E. Deniz, and D. Raducanu, “The Fekete-Szegö problem for subclasses of analytic functions defined by a differential operator related to conic domains," Computers \& Mathematics with Applications, vol. 59, no. 1, pp. 283-295, 2010.

[10] H. Orhan, E. Deniz, and M. Çağlar, "Fekete-Szegö problem for certain subclasses of analytic functions," Demonstratio Mathematica, vol. 45, no. 4, pp. 835-846, 2012.

[11] H. M. Srivastava, A. K. Mishra, and M. K. Das, "The FeketeSzegö problem for a subclass of close-to-convex functions," Complex Variables. Theory and Application, vol. 44, no. 2, pp. 145-163, 2001.

[12] P. Wiatrowski, "The coefficients of a certain family of holomorphic functions," Zeszyty Naukowe Uniwersytetu Lodzkiego Nauki Matematyczno Przyrodniczego Seria, no. 39, pp. 75-85, 1971.

[13] D. Răducanu and H. Orhan, "Subclasses of analytic functions defined by a generalized differential operator," International Journal of Mathematical Analysis, vol. 4, no. 1-4, pp. 1-15, 2010.

[14] G. Sălăgean, "Subclasses of univalent functions," in Complex Analysis - 5th Romanian-Finnish seminar, vol. 1013 of Lecture Notes in Mathematics, pp. 362-372, Springer, Berlin, Germany, 1983.

[15] F. M. Al-Oboudi, "On univalent functions defined by a generalized Sălăgean operator," International Journal of Mathematics and Mathematical Sciences, no. 25-28, pp. 1429-1436, 2004.

[16] G. Murugusundaramoorthy, "Subordination results for spirallike functions associated with the Srivastava-Attiya operator," 
Integral Transforms and Special Functions, vol. 23, no. 2, pp. 97103, 2012.

[17] O. S. Kwon and S. Owa, "The subordination theorem for $\lambda$-spirallike functions of order $\alpha$," Sūrikaisekikenkyūsho Kōkyūroku, no. 1276, pp. 19-24, 2002.

[18] H. Silverman, "Sufficient conditions for spiral-likeness," International Journal of Mathematics and Mathematical Sciences, vol. 12, no. 4, pp. 641-644, 1989.

[19] Z. Nehari, Conformal Mapping, McGraw-Hill, London, UK, 1952. 


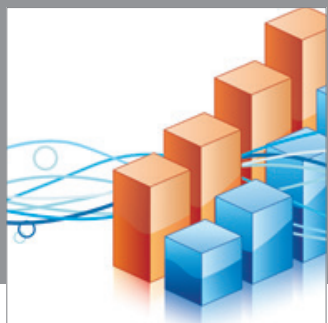

Advances in

Operations Research

mansans

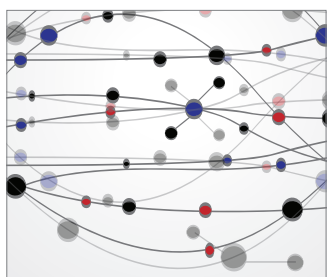

The Scientific World Journal
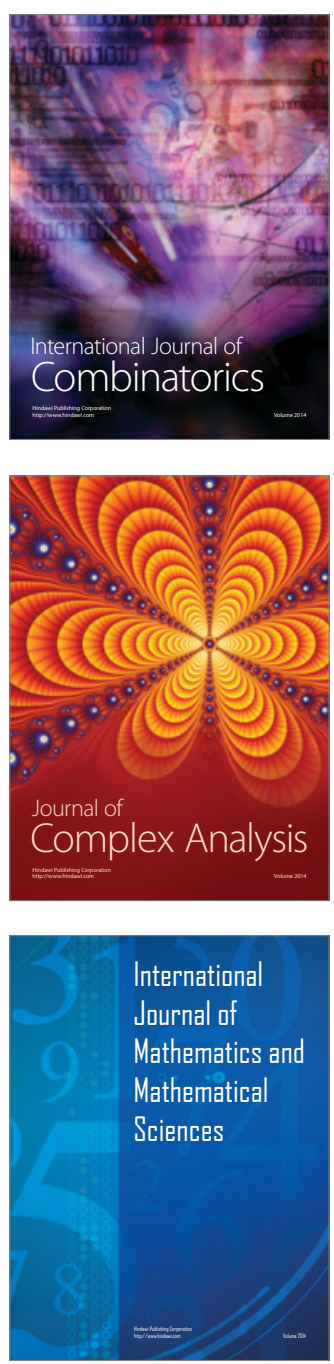
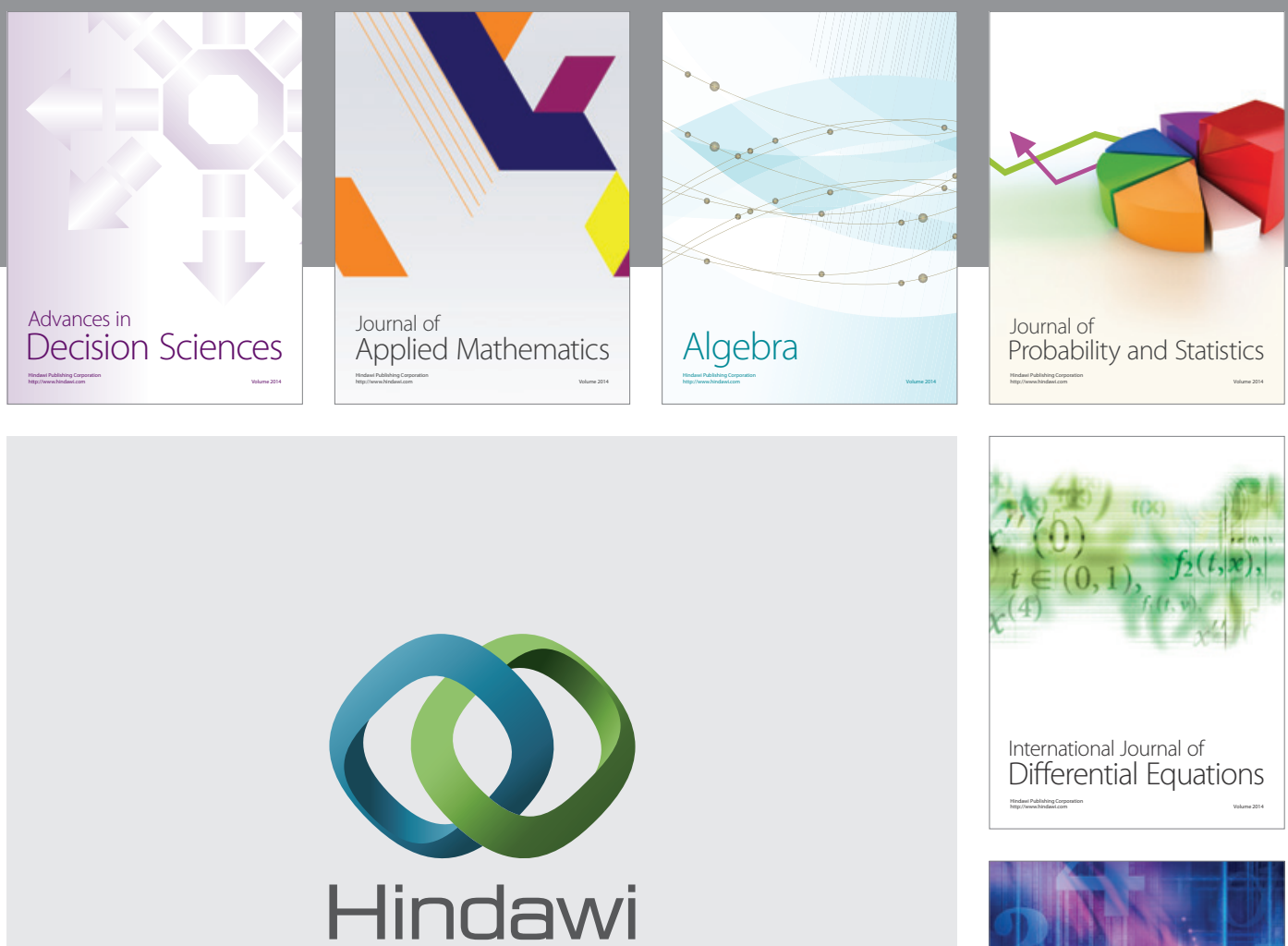

Submit your manuscripts at http://www.hindawi.com
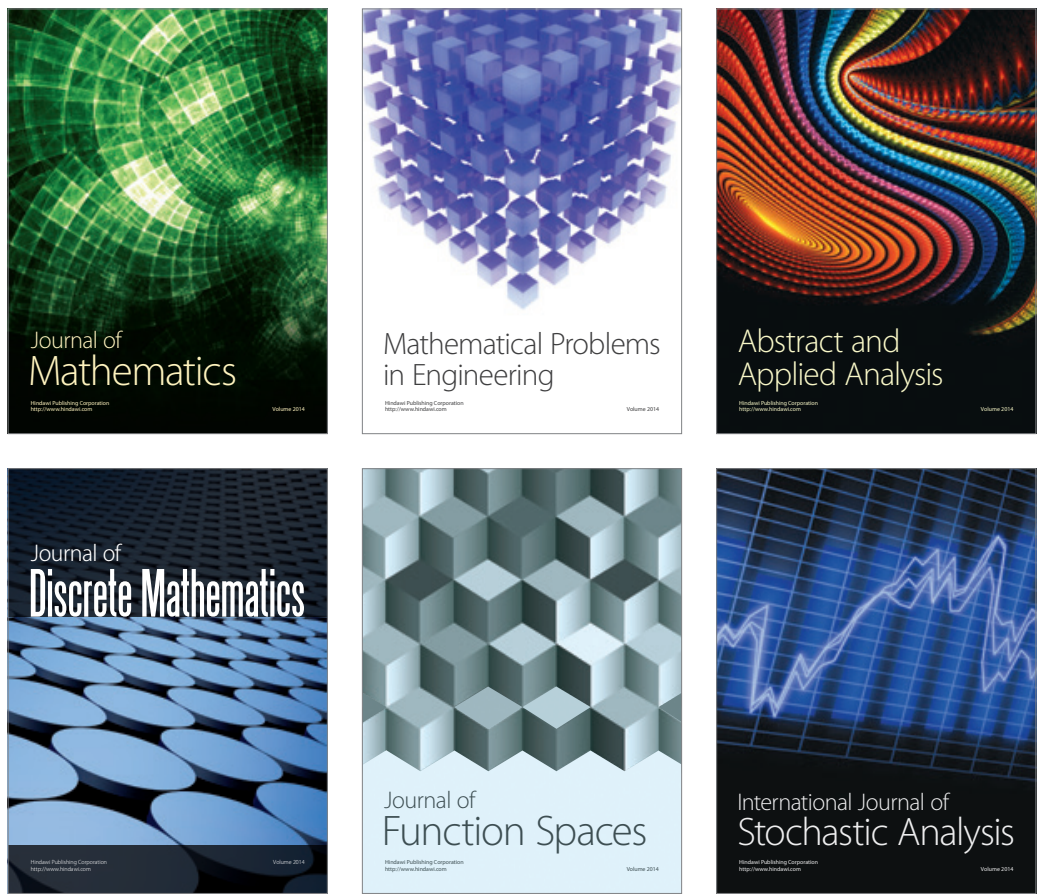

Journal of

Function Spaces

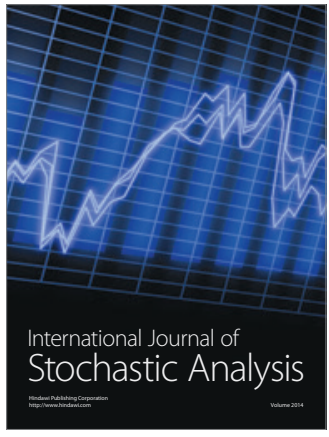

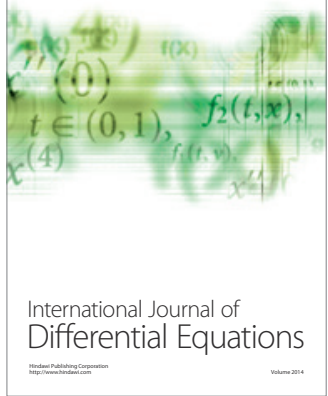
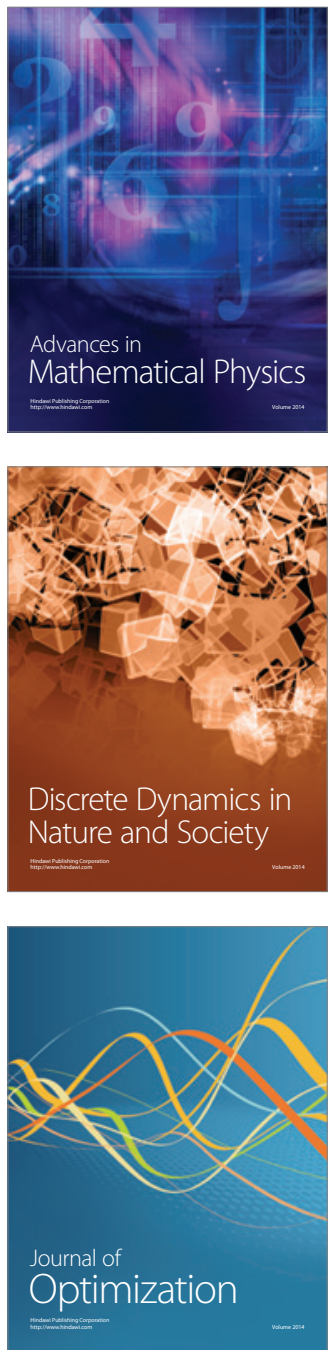